\title{
EL DESPLAZAMIENTO DE LOS PISOS TÉRMICOS Y EL \\ LENGUAJE SEMIÓTICO DE LAS PLANTAS COMO UNA \\ EXPRESIÓN DE SU ESTRÉS BIOLÓGICO: DOS \\ IMAGINARIOS SOCIALES DE LA POBLACIÓN CALDENSE \\ SOBRE LOS EFECTOS GENERADOS POR EL CAMBIO \\ CLIMÁTICO
}

\author{
YOLANDA AGUIRRE OSPINA ${ }^{1}$
}

Recibido el 16 de septiembre de 2012 y aprobado el 13 de noviembre de 2012

\section{RESUMEN}

Se presenta parte de los resultados obtenidos en una investigación de tipo cualitativo que tuvo como propósito develar los imaginarios sociales que la población caldense tiene sobre cambio climático; y al tiempo resaltar su importancia para convocar acciones participativas e incluyentes sobre cambio climático en el departamento de Caldas, pues, en tanto, esquemas construidos socialmente, son ellos los que estructuran y engendran comportamientos colectivos que se condensan en apreciaciones, gustos, ideales, valores; prácticas culturales; y como tales pueden constituirse en paradigmas sociales que según su grado de instauración posibilitan o bloquean los desarrollos de una región en sus distintas dimensiones.

En ese sentido, la población caldense a través de sus percepciones de cambios en el entorno permitió develar el desplazamiento de los pisos térmicos y el lenguaje semiótico de las plantas como dos imaginarios de los efectos generados por el cambio climático, que demandan emprender acciones de investigación sobre el grado de afectación y reconfiguración de los pisos térmicos y diseñar estrategias de mitigación y adaptación con base en la expresión del estrés hídrico manifestado por la vegetación ante los efectos generados por el cambio climático.

\section{PALABRAS CLAVE}

Imaginarios sociales, migración pisos térmicos, estrés hídrico de las plantas. 


\title{
DISPLACEMENT OF THERMAL FLOORS AND THE SEMIOTIC LANGUAGE OF PLANTS AS AN EXPRESSION OF THEIR BIOLOGICAL STRESS: TWO SOCIAL COLLECTIVE IMAGINATIONS OF THE CALDAS POPULATION ABOUT THE EFFECTS GNERATED BY CLIMATE CHANGE
}

\begin{abstract}
Part of the results obtained in a qualitative type research with the purpose of unveiling the social imaginations of the Caldas population about climate change are presented in this paper, and, at the same time, their importance to convene participative and inclusive actions about climate change in the Department of Caldas since schemas socially constructed structure and generate collective behaviors that condense in appreciations, ideals, values, and cultural practices which can constitute into social paradigms that, depending on their establishment degree, facilitate or block the development of a region in its different dimensions.

In this sense, the Caldas population, through their perceptions of change in the environment, allowed the unveiling of the displacement in the thermal floors and the semiotic language of plants as two collective imaginations of the effects generated by climate change which request the undertaking of research actions about the affectation and reconfiguration of thermal floors as well as the design of mitigation and adaptation strategies based on the expression of the hydrological stress manifested by vegetation before the effects generated by climate change.
\end{abstract}

\section{KEY WORDS}

Collective imaginations, displacement of thermal floors, plants hydrological stress.

\section{INTRODUCCIÓN}

Investigar sobre los imaginarios sociales, permitir que la gente los exprese y contribuir a una mayor democracia ambiental, a la coparticipación 'comunidad- instituciones varias -sectores encargados de la toma de decisiones', logrando que las personas conozcan y tomen su posición frente a las condiciones de su entorno y cómo ellas afectan sus condiciones de vida, su cotidianidad, su mundo, demanda indagar sobre la percepción de la gente, sus valores y expectativas frente al fenómeno del cambio climático, y desde allí, tratar de buscar una identidad propia sobre esta problemática y lograr "una reapropiación de la cultura y la naturaleza, desde la construcción de nuevos derechos colectivos y de otra racionalidad social - de una racionalidad ambiental- que siente las bases para un futuro sustentable" (Leef, 2010, p. 44). 
Mediante esta investigación se indagó sobre los cambios que la población caldense percibe en sus hábitos de vida y en su entorno. Son estas percepciones las que permiten captar las representaciones que tiene la población sobre cambio climático, para luego asignarle una interpretación o, mejor, un significado. Las percepciones en esencia constituyen "los instrumentos básicos mediante los cuales los imaginarios construyen algo como real" (Gómez, 2001, p. 204) y esos imaginarios construidos colectivamente, no son solo representaciones de un mundo o de una cosmovisión, ellos, según, Castoriadis, 1975 (citado en Leff, 2010, p. 85) están "arraigados en las identidades que conforman al ser cultural; se afirman como un principio de autonomía y de singularidad desde donde no sólo resisten a la colonización de otras culturas dominantes y hegemónicas, sino desde donde se resignifican sus identidades"; es a partir de esos imaginarios que la sociedad se interpreta y representa su realidad para poder intervenirla, modificarla, transformarla.

En ese sentido, se presenta el desplazamiento de los pisos térmicos y el lenguaje semiótico de las plantas como dos importantes imaginarios develados desde las percepciones que la población caldense tiene sobre cambio climático, y se constituyen en dispositivos para emprender acciones dirigidas a la mitigación de los impactos generados en nuestros ecosistemas y a la formulación de estrategias de adaptación a los efectos producidos por el cambio climático en nuestro entorno, tales como la migración de los pisos térmicos y los cambios en la vegetación; signos de estrés hídrico que hay que aprender a leer e interpretar de tal forma que puedan constituirse en indicadores de alerta de los efectos generados por el cambio climático, y objetos de simulación para diseñar estrategias de adaptación y mitigación de los daños generados por este fenómeno en nuestra región.

\section{METODOLOGÍA}

Se trabajo con base en las subregiones definidas para el departamento de Caldas: Oriente, Centro Oriente, Sur, Centro Sur, Occidente Alto, Occidente Bajo.

Para la recolección de la información se aplicaron 383 encuestas a integrantes grupos de trabajo vinculados a distintos programas adelantados por CORPOCALDAS, entre ellos: Ios consejos de desarrollo sostenibles y los consejos de cuencas, dada su pluralidad, representatividad cultural y social, por subregiones; a una población aleatoria conformada por estudiantes, agricultores, campesinos, amas de casa, madres comunitarias, entre otros. Así mismo, se realizaron 12 entrevistas semiestructuradas a alcaldes, secretarios de desarrollo o los delegados de las administraciones municipales y a representantes de instituciones encargadas de implementar acciones sobre cambio climático en el departamento de Caldas, 
y finalmente se desarrollaron tres conversatorios con grupos focales.

Para el análisis e interpretación de la información se utilizó el Excel y el Atlas ti; considerando necesario realizar un análisis estadístico descriptivo que permitiera identificar relevancias y opacidades derivadas de un primer tratamiento de información a través del software elegido para el análisis de la información recolectada (Atlas ti).

\section{RESULTADOS}

\section{El desplazamiento de los pisos térmicos}

El departamento de Caldas está situado en el centro occidente de la región andina, localizado entre los 05 $46^{\prime} 51^{\prime \prime}$ y los 04⒋'20"de latitud norte, y los 74038'01" y 75055'45" de longitud oeste. Cuenta con una superficie de $7888 \mathrm{~km}^{2}$, lo que representa el 0,69\% del territorio nacional. Fisiográficamente comprende áreas montañosas correspondientes a la cordilleras Central y Occidental; áreas suavemente onduladas ubicadas hacia las zonas de peneplanicies hasta su contacto con los valle medio del río Magdalena hacia el oriente y con los ríos Cauca y Risaralda hacia el occidente.

Esta ubicación geográfica y su diversidad fisiográfica le otorgan al departamento cualidades muy especiales entre las que se resalta: la posesión de los diferentes pisos térmicos desde el cálido hasta el glacial y la consecuente presencia de suelos fértiles y pluviosidad adecuada, lo cual le ofrece grandes posibilidades en términos de biodiversidad y aprovechamiento agropecuario de su territorio (ver Tabla 1).

La temperatura del departamento de Caldas varía de acuerdo con la altitud y el relieve y está influenciada por los vientos alisios del noreste y del sureste. Sobre el flanco oriental de la cordillera Central se localizan los sectores más lluviosos, entre los 1200 y 1600 metros de altura, donde la precipitación supera los $3000 \mathrm{~mm}$ anuales. Los sectores con menos de 1500mm anuales se ubican sobre los $3500 \mathrm{~m}$ de altura, en el parque nacional natural de los Nevados, que incluye las máximas alturas del departamento. La distribución de los pisos térmicos es cálido el $32 \%$ del total del departamento, templado $36 \%$, frío $23 \%$ y el piso bioclimático de páramo $9 \%$. 
Tabla 1. Pisos térmicos, extensión y temperaturas en el departamento de Caldas. Gobernación de Caldas, 1987 (CORPOCALDAS, 2005).

\begin{tabular}{|c|c|c|c|}
\hline $\begin{array}{c}\text { PISO } \\
\text { TÉRMICO }\end{array}$ & $\begin{array}{c}\text { ALTURA } \\
(\mathrm{msnm})\end{array}$ & $\begin{array}{c}\text { SUPERFICIE } \\
\left(\mathrm{km}^{2}\right)\end{array}$ & $\begin{array}{c}\text { RANGO DE } \\
\text { TEMPERATURA } \\
\left({ }^{\circ} \mathrm{C}\right)\end{array}$ \\
\hline Cálido & $0-1000$ & 2250 & $25-29$ \\
\hline Templado & $1000-2000$ & 2750 & $17-23$ \\
\hline Frío & $2000-3000$ & 1650 & $11-15$ \\
\hline Páramo & $3000-4000$ & 800 & $7-11$ \\
\hline Nieve (glacial) & 4000 y más & 57 & $5-0$ \\
\hline
\end{tabular}

El piso cálido se presenta hacia el oriente del departamento en los sectores de los ríos Magdalena y La Miel, así como en el occidente sobre el cañón del río Cauca y en el valle del Risaralda. El piso templado se encuentra en ambas vertientes de la cordillera Central y al oeste del río Cauca y corresponde a la zona cafetera. El piso de clima frío, el de páramo y la zona de nieves perpetuas se localiza en las partes más altas de la cordillera Central.

Las precipitaciones corresponden a condiciones tropicales con una alternancia de los periodos lluviosos (invierno) y secos (verano) y una intensidad que depende de la altitud y en algunos casos de las condiciones topográficas. Las precipitaciones son de tipo convectivo con dos periodos de máxima lluvia, uno de abril a mayo y otro de octubre a noviembre; julio por lo general es el mes más seco, excepto en La Dorada donde febrero y marzo suelen ser más secos. En el cañón del río Cauca se presenta también un largo periodo seco. La precipitación anual varía entre $1663 \mathrm{~mm}$ en Anserma y 3749,3 mm en Samaná (Gobernación de Caldas, 1987, citado por CORPOCALDAS, 2005).

Esta breve caracterización climática y fisiográfica del departamento de Caldas se refiere muy a propósito, dado que en esencia es retomada desde varios documentos que aparecen en internet y de algunos informes finales recientes (2005, 2006, 2007) elaborados por CORPOCALDAS en relación con el estado actual de los páramos en el departamento y su plan de manejo; el desarrollo de la fruticultura en Caldas adelantado por el Fondo Nacional de Fomento Hortifrutícola FNFH-, Asociación Hortifrutícola de Colombia -Asohofrucol-, Sociedad de Agricultores y Ganaderos del Valle del Cauca SAG-, (2006) entre otros; de esta manera se pretende establecer una pauta de comparación entre el conocimiento instituido en el colectivo de la población caldense y aquel emergente desde las percepciones y testimonios aportados por la población para esta investigación, que develan la migración de los pisos térmicos y la afectación de los ecosistemas. Es la población la que se expresa:

Neira es muy extenso, tiene 52 veredas y tiene todos los pisos térmicos [...] Manga Bonita que es la parte más fría del municipio anteriormente era productora de papa, producía mucha papa y era la base de la economía de esta gente; 
debido al cambio de clima las zonas para el cultivo de papa han tenido que irse subiendo, eso ha traído primero problemas en el ecosistema por la deforestación de áreas protegidas para siembra de papa, allá están todos los abastecedores de guanábana [...]. La gente al buscar tierras para sembrar papa ha comenzado a deforestar bosques primarios [...] (E-Neira 1).

[...]También se ven cambios en las temperaturas porque antes tierras que no eran aptas para cultivo de café ahora son aptas ya que las condiciones climáticas son totalmente diferentes, y las que eran más bajitas se volvieron más calientes y susceptibles a plagas y enfermedades (E-Anserma 1).

En el municipio se ha notado el cambio climático, primero la frontera agrícola se ha movido o sea la zona cafetera de este municipio ha venido ascendiendo, encontramos por ejemplo: el cultivo de café, anteriormente lo que era la vereda del Río, la vereda Cholo y parte de la vereda Armenia era eminentemente cafetera, en estos momentos debido al aumento de la temperatura media estas veredas han cambiado su vocación agrícola hacia la ganadería, hacia la floricultura; encontramos por ejemplo otras veredas de la parte más alta del municipio (la parte de Santa Isabel, lo que es el Jardín, lo que es Pueblo Viejo) que eran zonas eminentemente ganaderas y para el cultivo de hortalizas, en este momento están dedicadas al cultivo de productos de clima más medio como lo es el plátano y café (E-Neira 1).

Se sienten muchos cambios, entre ellos está el desplazamiento de los cultivos, por ejemplo nosotros teníamos ubicado en la parte baja del municipio unos cultivos que están a más o menos $450 \mathrm{msnm}$ que nos ha tocado cambiarlos, nosotros allá teníamos café el cual se ha desplazado a la zona alta y allá tocó remplazarlo por cítricos y pastos porque el café ya no nos da allá, con las plagas no somos capaces en esa zona; entonces a través del entorno se ha observado eso, los cultivos han subido; por ejemplo en la parte marginal superior que está ubicada a $1800 \mathrm{~m}$ ya tenemos allá café y antes allá no nos daba café; entonces el comportamiento de los cultivos ha cambiado (E-Risaralda 2). 
Son varias las voces, varios los municipios, pero todos ellos hacen referencia al desplazamiento de los cultivos y al incremento de la temperatura de tal forma que las condiciones de los terrenos aptos, tiempos atrás, para ciertos cultivos, por ejemplo el café y la papa ya no son aptos en la actualidad, implicando el desplazamiento o migración de los pisos térmicos tradicionalmente definidos y su concomitante afectación a los ecosistemas caldenses, nuestros ecosistemas. La Figura 1 esquematiza lo referido, sugiriendo la presión permanente que ejerce el hombre sobre los distintos ecosistemas para colonizar nuevas tierras y desarrollar actividades agrícolas y pecuarias.

Se ven afectaciones en las partes bajas; por los calores ya no nos sirve para cultivar el café y han tenido que recurrir a otra clase de cultivos que no son tan rentables como el café; y en las partes altas lógicamente las lluvias, los deslizamientos, la escasez en el agua; lógicamente los más afectados son la zona rural (E-Belalcázar 1).

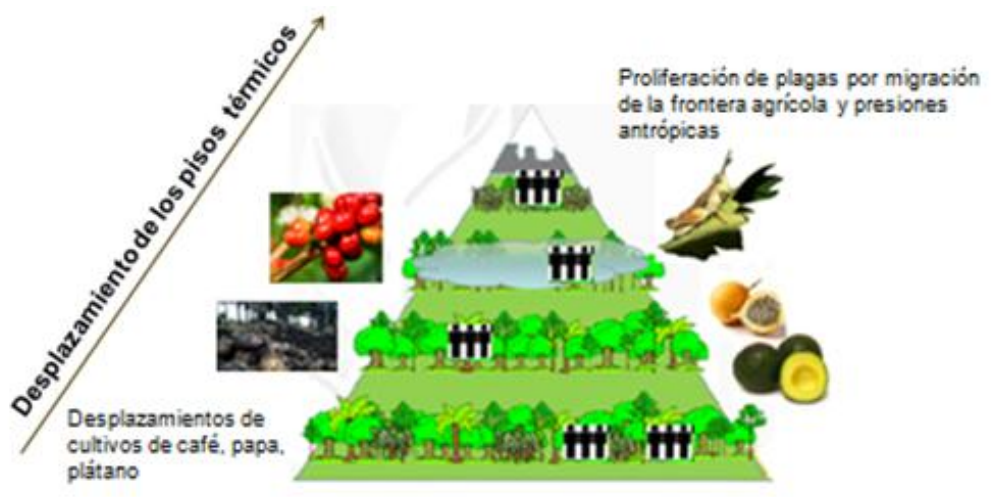

Figura 1. Desplazamiento de los pisos térmicos y algunos de sus indicadores en el departamento de Caldas (modificado y adaptado de Matallana, 2010).

Los testimonios también evidencian cómo la presión de las actividades del hombre afecta negativamente la estabilidad de los ecosistemas, intensificando procesos erosivos, afectando las zonas de protección y la capacidad regenerativa de la tierra:

[...] esta es una zona eminentemente ganadera, el principal problema es que la ganadería a esa altura produce compactación del suelo, produce erosión, y entonces como la regeneración del pasto es muy lenta empieza a atacar las zonas que son de protección (ENeira 1).

[...] se ha visto por ejemplo alguna variedad de grillos. Aquí anteriormente se veían en determinada época, ahora ya han permanecido mucho más tiempo, y ese tipo de plagas afectan también los cultivos [...] se está volviendo a presentar y curiosamente aquí, los 
dos tipos de plaga popular del café, la roya y el otro gorgojito, se me escapa el nombre [...] (ESupía 1).

[...] las prácticas normales de control con químicos o controles biológicos naturales, no han tenido su trascendencia o su importancia, porque muchas plagas han generado resistencia a esos mismos químicos; en la parte biológica se han hecho diferentes esfuerzos, por ejemplo con el gusano que afecta directamente los cultivos de caña panelera; hemos notado que ese gusano, que en últimas es una mariposa ya ha empezado a mutar y a generar niveles de resistencia en una proporción inmensa; otro bichos que son populares en esta zona pero que no tienen tanta injerencia, como la hormiga, la hormiga arriera que solamente se tenía identificado unos pequeños focos, ahora se ha propagado en una gran extensión [...] (E- Supía 1).

Como se puede observar, la población caldense identifica dispersión y proliferación de plagas, especies invasoras que pueden afectar notablemente la composición de los ecosistemas y la propia seguridad alimentaria al causar daños severos en los cultivos.

\section{El lenguaje semiótico de las plantas: una expresión de su estrés biológico}

Al indagar a la población caldense sobre los cambios percibidos en la vegetación, se pudo detectar la sensibilidad de las personas ante los cambios en la coloración, el brillo de las hojas, el crecimiento de las plantas y el grado de marchitación, entre otros; manifestando apreciaciones como: la vegetación 'se nota cada vez más marchita, más opaca'; 'su verde ya no es natural', 'cada vez es menos verde'; se observa 'amarillamiento de las hojas'.

Todas estas expresiones no son más que lecturas semióticas de los signos cotidianos que emite la vegetación y que en esencia nos pueden permitir elaborar un diagnóstico epidemiológico de su patología, el grado de afectación de sus dinámicas como resultado de sus interacciones con factores ambientales tales como cambios excesivos en el clima, cambios en la temperatura (percepciones de alta relevancia dentro de la población caldense) y de la intervención antrópica, situaciones que se traducen en la disminución de las cosechas y la afectación negativa del ciclo regenerativo y de vida de la vegetación.

Hsiao (1973) y Levitt (1980) (citados en Valladares et al., 2004), dicen que una planta está sometida a estrés cuando se ve expuesta a condiciones significativamente diferentes de las óptimas para su desarrollo, señalando además que los 
requerimientos óptimos difieren según las distintas especies y variedades; variando además su susceptibilidad a un estrés determinado; Levitt (citado en Hernández, 2011, p. 166) afirma que "cualquier factor ambiental es capaz de producir una deformación (strain) potencialmente nociva en un organismo".

Cuando la población refiere que la vegetación 'muere por excesivos cambios del clima', 'se afecta por la temperatura' o las 'cosechas ya no se dan en las fechas adecuadas', están evidenciando que las condiciones 'tradicionales' o 'normales' para garantizar el desarrollo de las cosechas y la vida de la vegetación han cambiado y reconocen la temperatura y los cambios extremos del clima como las principales causas de esos cambios. Ante lo expresado, resulta muy oportuno acotar que las variables climáticas de interés en estudios epidemiológicos son precisamente: la temperatura, la humedad, la pluviosidad, la radiación y el viento (Campell \& Madden, 1990, citados en Hernández, 2011).

Según varios autores, entre ellos Vale et al. (2004) y Hernández (2005) (citados en Hernández, 2011, p.11), la temperatura como tal "influye en los procesos de infección, colonización, esporulación, sobrevivencia del patógenos y también en los procesos fisiológicos de la planta, como fotosíntesis, evapotranspiración, metabolismo entre otros", así mismo y solo por ejemplificar la influencia de otra de las variables climáticas en el óptimo desarrollo de los plantas es pertinente mencionar la importancia de la humedad (ya sea la humedad del suelo, la humedad relativa, las precipitaciones o el mojamiento foliar) para el proceso de germinación de semillas (Hernández, 2011).

Los estudios realizados por varias entidades -entre ellas Cenicafé, el INCADES, la Corporación Colombiana de Investigación Agropecuaria (Corpoica), varias universidadeshan abordado, para el caso del café, el cacao, la mandarina, el frijol, la granadilla, entre otros, el grado de afectación que el aumento de temperatura ha producido en estos cultivos, en las últimos años (periodos que alcanzan hasta las últimas décadas), y han podido identificar cambios en sus ciclos de vida, su fisiología, su calidad, su patogénesis y su rendimiento. Son los testimonios de la población caldense los que ponen en evidencia algunos de esos cambios cuando manifiestan:

[...] las épocas que nosotros considerábamos sin lluvias se corren o hay exceso de lluvias, eso afecta producciones; inclusive la ubicación de ciertos cultivos en algunas zonas se han visto afectadas; ya no es fácil decir que bajo esta altura por ejemplo podemos sembrar este tipo de alimentos porque se nos están dando exceso de humedad y agua, entonces ya se vuelve muy complicado [...] (E-Pensilvania 2).

[...] con exceso de lluvia las floraciones se pierden; en el tema específico del café, las floraciones están supremamente afectadas, yo 
creo que desde hace mucho tiempo no hay la cantidad que debiera haber (E-Pensilvania 2).

[...]Belalcázar cambio de clima antes frío y húmedo; pero ahora vemos un aumento de la temperatura, ahora tenemos unos calores hasta insoportables; de hecho en las zonas bajas del municipio que ya no son aptas para el cultivo del café. Antes el cultivo predominante era el café; ahora las partes bajas solo sirven para cultivar caña y para pastos; en las partes más altas se está cultivando café y plátano (EBelalcázar 1).

El sector más afectado fue espárragos, muy difícil de solucionar, porque con la ola invernal sí se afecta la parte productiva porque uno los puede ver muy bonitos, pero lo importante es la parte de abajo que es donde le da el hongo; café y plátano en la medida que la productividad disminuye. Uno ve el cambio de clima ahora, estos calores que agotan lo que sea y cuando llega la noche son heladas; pasa lo de la sabana de Bogotá [...] (E-Anserma 1).

Generalmente en esta zona es el exceso de aguas, no sequías; el café no se madura correctamente, se seca verde y es un café de mala calidad, no se vende casi, afectando la parte económica; el café es la base en toda la parte oriente prácticamente; el tema del cacao por estas lluvias es exactamente el mismo [...] (E-Pensilvania 2).

Se han afectado las producciones de los cultivos debido a que se caen las floraciones, se queman las floraciones como en el caso del café, se caen las producciones biches, entonces todo eso afecta obviamente el sistema productivo, además el tema de enfermedades es extremadamente extraño [...] (E-Manzanares 2).

[...] los cultivos han cambiado, o sea, hace cuatro años hubo un boom con la cosecha de granadilla, llegamos a tener 750 hectáreas de granadilla; hoy en día no creo tener la décima parte de eso; llegó un hongo que produce la secadera, que obviamente lo favoreció las lluvias [...] (E-Aránzazu 1).

En el café se ha disparado la roya por ejemplo y la broca, que son pues plagas típicas del café, han tenido que hacer mayor inversión en los fungicidas, igualmente en el plátano, ya que aquí se ha estado diversificando con el plátano 
por el problema que ha tenido el café por estas dos plagas; pero obviamente en el plátano surgen plagas por la variación en el clima; y principalmente se quejan de estas plagas que surgen gracias a el clima tan cambiante. Años atrás habían meses que se sabía fijo cuándo llovía o era verano, hoy en día ya desapareció y son impredecibles los climas (E- Belalcázar 1).

Desde los testimonios se puede observar que la variabilidad climática con su aumento de temperatura y sus consecuentes oleadas de calor extremo, o los periodos de lluvias intensas, han generado estrés biológico en cultivos como el café, la granadilla, el plátano, el frijol, el tomate de cocina, evidenciado en la variación de los ciclos de producción, las floraciones, los procesos de fotosíntesis de los cultivos, proliferación de plagas, entre otros.

Es importante señalar que de las situaciones de estrés que pueden llegar a afectar los diferentes cultivos, una de las más estudiadas es el estrés hídrico. Son muchos los documentos que se pueden consultar con respecto a investigaciones epidemiológicas adelantadas para identificar factores climáticos y su influencia en la ocurrencia, variación o severidad de las enfermedades que sufren las plantas y ante los resultados obtenidos y la alerta expresada por la población caldense es perentorio otorgar una mayor atención a esta problemática y promover la generación y desarrollo de proyectos a corto y mediano plazo, de tal forma que se puedan identificar especies potenciales a la adaptación de los cambios climáticos proyectados por el IPCC (2007) y por la Segunda Comunicación Nacional sobre Cambio Climático; adoptar políticas y estrategias para reducir su impacto en el futuro y contribuir a vislumbrar un desarrollo sustentable para nuestras regiones.

El estrés hídrico es la tensión generada sobre la vegetación por el déficit de agua, por las sequías, como tal "se produce en las plantas en respuesta a un ambiente escaso en agua, en donde la tasa de transpiración excede a la toma de agua" (Moreno, 2009, p. 180). Es muy importante señalar, como bien lo subrayó Levitt, 1980 (citado por Moreno, 2009, p. 181), "que el déficit hídrico no solo ocurre cuando hay poca agua en el ambiente, sino también por bajas temperaturas y por una elevada salinidad del suelo".

En los sistemas naturales, un déficit de agua puede ser el resultado de bajas precipitaciones, baja capacidad de retención de agua del suelo, excesiva salinidad, temperaturas extremas frías o calientes, baja presión de vapor atmosférica o una combinación de estos factores (Nilsen \& Orcutt, 1996, citados en Moreno, 2009, p. 181)

Hanson y Hitz, 1982, citados en Moreno, 2009, p. 181) reconocen el agua, como el "principal factor limitante del crecimiento de las plantas en la tierra, actuando como una 
fuerza selectiva de primer grado para la evolución y distribución de las especies vegetales".

Las plantas expuestas a condiciones de estrés hídrico generan mecanismos de respuesta que pueden ser fácilmente detectados por el hombre, estas respuestas pueden evidenciar cambios o mecanismos desarrollados por ellas para evitar ese estrés, tolerarlo o adaptarse. El conocimiento de los mecanismos de resistencia al estrés permite comprender los procesos evolutivos implicados en la adaptación de las plantas a ambientes adversos, y predecir hasta cierto punto la respuesta vegetal al incremento de la adversidad asociada en muchos casos al cambio global. Además, pueden ser aplicados para mejorar las características de las plantas tanto en su fase de cultivo como en la selección de variedades que se ajusten a unos requerimientos ambientales determinados o, simplemente, en mejorar la productividad de una especie [Kozlowski et al. (1991) y Nilsen \& Orcutt, citados en Valladares et al. (2004)].

El estudio de las respuestas de las plantas al estrés es un aspecto fundamental de la fisiología ambiental o ecofisiología, la cual se propone conocer cómo las plantas funcionan en sus ambientes naturales y cuáles son los patrones que determinan su distribución, supervivencia y crecimiento [Kramer \& Boyer (1995), Lambers et al. (1998) y Ackerlyet al. (2002) citados en Valladares (2004)].

Investigaciones realizadas en Perú sobre el café han mostrado que el cambio climático afecta tanto la fisiología de los cultivos como la biología del patógeno determinando que en un futuro las zonas altas no escaparán a brotes epidémicos, y la severidad de la roya del cafeto no será tan baja como en los años 80; además, encontraron que la broca del cafeto se presenta en alturas mayores que las tradicionales; antes solo afectaba cafetales por debajo de 1200msnm y ahora se encuentra en cultivos por encima de los $1500 \mathrm{msnm}$, aspecto que está relacionado con el incremento de la temperatura (Hernández y Montoya \& Ríos, citados en Hernández, 2011). Siguiendo a este último autor:

[...] debido a los cambios de temperatura que va en aumento, la calidad del café, también va a disminuir drásticamente. Viendo prospectivamente los escenarios, si las temperaturas aumentan en $3^{\circ} \mathrm{C}$ hasta el final de este siglo, se puede inferir que la buena calidad de café arábigo, tal como se cultiva en las regiones andino amazónicas, se conseguirá desplazando el cultivo a una "velocidad" de aproximadamente $50 \mathrm{msnm}$ por década. Esto provocará plantar cafetales en alturas mayores a los 1800 o $1900 \mathrm{msnm}$, donde nacen las fuentes de agua de la selva alta, causando serios daños ecológicos (Hernández, 2011, p. 15). 
Por su parte, Sisó et al. 2001, (citados en Valladares et al., 2004, p.181) han mostrado cómo la forma de las hojas de ciertas especies puede ser un indicador de las condiciones hídricas de la planta, ellos observaron que la mayor lobulación de las hojas de distintas especies de robles caducifolios implica una mejora hídrica dado que las hojas más lobuladas tienen una menor resistencia hidráulica.

Considerando que el estrés biológico hace referencia a cualquier factor ambiental capaz de producir un signo no común, una alerta, una deformación potencialmente nociva en una planta, es necesario realizar estudios de la patología en poblaciones de plantas y en sus dinámicas de tal forma que se puedan identificar especies o grupos de especies con un comportamiento ecológico o fisiológico similar que permita predecir los posibles cambios asociados al cambio climático y a la variabilidad climática. Parafraseando a Kozlowski et al.(1991) y Nilsen y Orcutt, (1996) (citados en Valladares et al., 2004): el conocimiento de los mecanismos de resistencia al estrés permitirán comprender los procesos evolutivos implicados en la adaptación de las distintas especies de plantas a un determinado ambiente y predecir en cierto grado, la respuesta de la vegetación a esas nuevas condiciones ambientales. Haciendo eco de lo ya dicho por Valladares et al. (2004, p. 180): "La incertidumbre radica una vez más en predecir qué especies desaparecerán y cuáles podrían volverse dominantes en los nuevos escenarios climáticos".

\section{CONCLUSIONES}

Develar los imaginarios sociales, comprenderlos como esa "fuente de lucidez y creatividad capaz de movilizar la energía social para deconstruir en el pensamiento y combatir en la práctica política la racionalidad insustentable" (Leff, 2010, p.52), puede ser el mejor camino para emprender acciones interinstitucionales e intersectoriales, incluyentes y participativas sobre los efectos que en nuestra región viene generando el cambio climático.

La ya presente migración de los ecosistemas demanda realizar estudios cartográficos integrales que permitan modelar su nueva distribución geográfica y permitan identificar sus cambios en distribución espacial; cambios en los servicios que los ecosistemas suministran a la población (agua, alimentación, recursos madereros, entre otros), cambios en la distribución espacial de flora y fauna, cambios en el patrón de ciclos biológicos y su relación con la temperatura y la disponibilidad de recurso hídrico (épocas de floración, reproducción, migración, productividad, etc.); solo así, se dará un paso adelante para generar modelos de respuesta propios, locales y regionales, ante el cambio climático y la variabilidad climática.

La variabilidad climática con su aumento de temperatura y sus consecuentes oleadas de calor extremo, o los periodos de 
lluvias intensas, han generado estrés biológico en cultivos como el café, la granadilla, el plátano, el frijol, el tomate de cocina, evidenciado en la variación de los ciclos de producción, las floraciones, los procesos de fotosíntesis de los cultivos, proliferación de plagas, entre otros.

\section{BIBLIOGRAFÍA}

- CORPOCALDAS y Conservación Internacional Colombia. (2005). Estudio sobre el estado actual de los páramos del Departamento de Caldas. Recuperado el 24 de mayo de 2011 de:http://www.paramo.org/files/recursos/Estado_Actual_ de_Paramos_de_Caldas.pdf

- CORPOCALDÁS. (2006). El papel de Corpocaldas como autoridad ambiental dentro de la gestión local del riesgo. Taller Internacional sobre Gestión del Riesgo a nivel local. El caso de Manizales, Colombia. Recuperado el 18 de abril de 2010 de:http://www.manizales.unal.edu.co/gestion_riesgos/d escargas/gestion/ CorpocaldasGestion.pdf

- . (2007). Plan de manejo de los páramos del departamento de Caldas. Informe Final.

- Gómez, P. A. (2001). Imaginarios Sociales y análisis semiótico. Una aproximación a la construcción narrativa de la realidad. Cuadernos, 17, 195-209. San Salvador: Universidad de Jujuy, Facultad de Humanidades y Ciencias Sociales, Secretaría de Ciencia y Técnica y Estudios Regionales.

- Hernández, T. A. (2011). Cambio climático y prospectiva epidemiológica sanitaria de los cultivos de café y cacao en la región Andino Amazónica. INCADES (Instituto de Asesoría y capacitación para el Desarrollo Sostenible). Recuperado el 25 de febrero de 2012 de: http://www.monografias.com/trabajos87/cambioclimatico-y prospectiva-sanitaria-cultivos-cafe-y-cacao/cambioclimatico -y-prospectiva-sanitaria-cultivos-cafe-y-cacao.shtml

- IPCC. (2007). Cambio climático: Informe de síntesis. Contribución de los Grupos de trabajo I, II y III al Cuarto Informe de Evaluación del Grupo Intergubernamental de Expertos sobre el Cambio Climático [Equipo de redacción principal: Pachauri, R.K. y Reisinger, A. (directores de la publicación)]. Ginebra, Suiza: IPCC.

- Leff, E. (2010). Imaginarios sociales y sustentabilidad. Seminario permanente de Cultura y Representaciones Sociales. Revista Electrónica html, 9. Recuperado el 28 de abril de 2011 de: http://www.culturayrs.com/?q=node/77

- Matallana, C. L. (2010). Cambio Climático y Biodiversidad: avances en Colombia. Bogotá: Instituto Humboldt. 
de:http://www.slideshare.net/InfoAndina/clara-

matallana-cambio-climtico-y-biodiversidad-junio20

- Moreno, L. P. (2009). Respuesta de las plantas al estrés por déficit hídrico. Una revisión. Revista Agronomía Colombiana, 27(2), 179-191.

- República de Colombia. Ministerio de Agricultura y Desarrollo Rural -MADR-, Gobernación de Caldas, Fondo Nacional de Fomento Hortifrutícola -FNFH-, Asociación Hortifrutícola de Colombia -Asohofrucol- y Sociedad de Agricultores y Ganaderos del Valle del Cauca -SAG-. (2006). Plan frutícola nacional. Desarrollo de la fruticultura en Caldas. Recuperado el 10 de enero de 2012 de: http://www.asohofrucol.com.co/archivos/bibliotecal biblioteca_101_Pan\%20Frut\%20CALDAS.pdf

- Valladares, F.; Vilagrosa, A.; Peñuelas, J.; Ogaya, R.; Camarero, J.; Corcuera, L.; Sisó, S. y Gil- Pelegrín, E. (2004). Estrés hídrico: ecofisiología y escalas de la sequía. En F. Valladares (Ed.), Ecología del bosque mediterráneo en un mundo cambiante (pp. 163-190). Madrid: Ministerio de Medio Ambiente, EGRAF, S.A.

1. Docente, Departamento de Ciencias Geológicas, Universidad de Caldas. yoly@ucaldas.edu.co 